\title{
ALTERNATOR AND ASSOCIATOR IDEAL ALGEBRAS
}

BY

\author{
IRVIN ROY HENTZEL $\left({ }^{1}\right)$, GIULIA MARIA PIACENTINI CATTANEO $\left({ }^{2}\right)$ AND

\section{DENIS FLOYD}

\begin{abstract}
If $I$ is the ideal generated by all associators, $(a, b, c)=(a b) c-$ $a(b c)$, it is well known that in any nonassociative algebra $R, I \subseteq(R, R$, $R)+R(R, R, R)$. We examine nonassociative algebras where $I \subseteq(R, R$, $R)$. Such algebras include $(-1,1)$ algebras, Lie algebras, and, as we show, a large number of associator dependent algebras. An alternator is an associator of the type $(a, a, b),(a, b, a),(b, a, a)$. We next study algebras where the additive span of all alternators is an ideal. These include all algebras where $I=(R, R, R)$ as well as alternative algebras. The last section deals with prime, right alternative, alternator ideal algebras satisfying an identity of the form $[x,(x, x, a)]=\gamma(x, x,[x, a])$ for fixed $\gamma$. With two exceptions, if this algebra has an idempotent $e$ such that $(e, e, R)=0$, then the algebra is alternative. All our work deals with algebras with an identity element over a field of characteristic prime to 6 . All our containment relations are given by identities.
\end{abstract}

Introduction. In a nonassociative algebra $R$, the associator $(a, b, c)$ for $a, b$, $c$ elements of $R$ is defined by $(a, b, c)=(a b) c-a(b c)$. Clearly $R$ is associative if and only if $(a, b, c)=0$ for all $a, b, c$ elements of $R$. If we let $I$ be the ideal of $R$ generated by all associators, then it is well known that $I \subseteq(R, R, R)+R(R, R, R)$. We examine algebras over a field $F$ of characteristic not 2 or 3 which have the property that $I \subseteq(R, R, R)$. We, however, assume more than the condition that the additive span of associators is an ideal. We assume that there is an absorption formula of the form

$$
a(b, c, d)=\sum_{\pi \in S_{4}} \lambda_{\pi}(a b, c, d)_{\pi}+\mu_{\pi}(a, b c, d)_{\pi}+\nu_{\pi}(a, b, c d)_{\pi} .
$$

The constants $\lambda_{\pi}, \mu_{\pi}$, and $\nu_{\pi}$ are elements of $F$; the subscript $\pi$ following the associator means that the unknowns of the expression are to be permuted by the permutation $\pi$. The terms of the summations include all possible ways that the original four elements can be arranged in an associator. We show that Lie

Received by the editors June 23, 1975.

AMS (MOS) subject classifications (1970). Primary 17A30; Secondary 17E05.

Key words and phrases. Associator ideal, right alternative, associator dependent.

( ${ }^{1}$ This work was done while the author held a Science and Humanities Research Institute grant from Iowa State University.

${ }^{(2)}$ This work was done while the author was at Iowa State University with a fellowship from the Consiglio Nazionale delle Ricerche, Italy. 
algebras and $(-1,1)$ algebras are examples of algebras satisfying such a formula; we show that alternative algebras with an identity element will never satisfy one (unless they are associative). We call condition (1) the "associator ideal" condition and the algebras which satisfy it "associator ideal" algebras. We show that with one exception, any algebra of the $(-1,1)$ type is an associator ideal algebra. We also give an example of an algebra in which the associators form an ideal but which is not an associator ideal algebra.

We extend our concept of associator ideal algebras to alternator ideal algebras. An alternator is an associator of the form $(a, a, b),(a, b, a),(b, a$, $a$ ). If we let $J$ be the ideal of a nonassociative algebra $R$ generated by all alternators, we examine algebras where $J$ is the additive span of alternators. Again, we assume that the algebra satisfies a specific formula that allows for the absorption of elements outside alternators into alternators. There are three types of alternators. Each type of alternator requires a formula for absorbing multiplication on the right side and on the left side. Any absorption formula needs six parts. In this paper we avoid such a general formula, but to display the generality and complexity, we give the identity for the absorption of $a(b, b, c) \in J$. The algebra is over a field $F$ of characteristic not 2 and not 3; we can assume a linearized identity. Let $M(x, y, z), M^{\prime}(x, y, z)$, and $M^{\prime \prime}(x, y, z)$ be defined as follows. $M(x, y, z)=(x, y, z)+(y, x, z)$; $M^{\prime}(x, y, z)=(x, y, z)+(z, y, x) ; M^{\prime \prime}(x, y, z)=(x, y, z)+(x, z, y)$. This part of the formula can then be written as follows:

$$
\begin{aligned}
a M(b, c, d)= & \sum_{\pi \in S_{4}} \alpha_{\pi} M(a b, c, d)_{\pi}+\sum_{\pi \in S_{4}} \beta_{\pi} M^{\prime}(a b, c, d)_{\pi} \\
& +\sum_{\pi \in S_{4}} \gamma_{\pi} M^{\prime \prime}(a, b c, d)_{\pi}+\sum_{\pi \in S_{4}} \delta_{\pi} M(a, b, c d)_{\pi} \\
& +\sum_{\pi \in S_{4}} \varepsilon_{\pi} M^{\prime}(a, b c, d)_{\pi}+\sum_{\pi \in S_{4}} \eta_{\pi} M^{\prime \prime}(a b, c, d)_{\pi}
\end{aligned}
$$

In the identity $\alpha_{\pi}, \beta_{\pi}, \gamma_{\pi}, \delta_{\pi}, \varepsilon_{\pi}, \eta_{\pi}$ are elements of the field $F$; the subscript permutation $\pi$ means that the four arguments are to be permuted by $\pi$.

We call these algebras "alternator ideal algebras". We show that every associator ideal algebra with identity is actually an alternator ideal algebra as well.

A right alternative alternator ideal algebra is a generalization of both $(-1$, 1) algebras and alternative algebras. If such an algebra $R$ is prime and has an idempotent $e \neq 0, \neq 1$ such that $(e, e, R)=0$, then with two possible exceptions the algebra is alternative.

Notation. All of our work assumes that we have an algebra over a field $F$ of characteristic prime to 6 so that scalar factors of the form $\frac{1}{3}$ and $\frac{1}{2}$ are admissible. 
As is common in algebra, we often place sets of elements in expressions where elements should appear. This is to be interpreted as the span of all things generated when the arguments for the expression are chosen from the indicated sets. Thus $(R, R, R)=$ the vector space over $F$ spanned by all $(a, b$, c) for $a, b, c \in R$.

We use both juxtaposition and "." to indicate multiplication. In expressions where both appear, the product indicated by juxtaposition is to be taken first; $(a, b, c)=a b \cdot c-a \cdot b c$.

If $R$ is any algebra, for any $a, b \in R$, we define "。" and "[ ]" by: $a \circ b=a b+b a ;[a, b]=a b-b a$. We indicate $(R,+, \circ)$ by $R^{+}$and $(R$, $+,[])$ by $R^{-}$. We use $(a, b, c)^{+}$to mean $(a, b, c)^{+}=(a \circ b) \circ c-$ $a \circ(b \circ c)$.

The words "identity" and "formula" refer to equations which hold for all elements in the ring. The "identity element" is the multiplicative identity 1 such that $1 \cdot a=a \cdot 1=a$ for all elements $a$.

In this paper all containment relations are by formula. We emphasize this by the symbol $\subseteq_{f}$. Thus, we write $R(R, R, R) \subseteq_{f}(R R, R, R)+(R, R R$, $R)+(R, R, R R)$, and we mean that there exists a specific formula of the form (1) which holds for all elements in $R$.

If $I$ is an ideal of an algebra $R$ and $I^{2}=0$, we call $I$ a trivial ideal. $R$ is called semiprime if $R$ has no nonzero trivial ideals. $R$ is called prime if whenever $I$ and $J$ are ideals such that $I J=J I=0$, then $I=0$ or $J=0$.

$S_{n}$ refers to the symmetric group of permutations on $n$ objects. Permutations refer to the positions, not the elements. Thus $\pi=(123)$ acting on $a, b, c$ is $c, a, b . \pi$ acting on $x_{1}, x_{2}, x_{3}$ is $x_{3}, x_{1}, x_{2} ; \pi$ acting on $x_{1}, x_{2}, x_{3}$ is not $x_{1 \pi}$, $x_{2 \pi}, x_{3 \pi}=x_{2}, x_{3}, x_{1}$. The subscript $\pi$ which appears in formulas like (1) means that the arguments are to be permuted by $\pi$. For example, if $\pi=$ (123)(4) $\in S_{4}$, then by $(a b, c, d)_{\pi}$ for $a, b, c, d \in R$, we mean $(a b, c$, d) $)_{\pi}=(c a, b, d)$.

The following identity is called the Teichmüller identity. It holds in any nonassociative algebra and may be verified by expansion.

$$
(a b, c, d)-(a, b c, d)+(a, b, c d)=a(b, c, d)+(a, b, c) d .
$$

In a right alternative algebra, the following identities hold; see [7, equations (2) and (3)].

(3) $(a, b, b c)=(a, b, c) b, \quad\left(a, b, b^{2}\right)=0, \quad\left(a, b, c^{2}\right)=(a, b c+c b, c)$.

Examples of associator ideal algebras. A Lie algebra is a nonassociative algebra satisfying the identities $a^{2}=0$ and $(a b) c+(b c) a+(c a) b=0$. In such an algebra, $(a, b, c)=(a b) c-a(b c)=(a b) c+(b c) a=-(c a) b$. Thus $(R, R, R)=R^{3}$ is an ideal of $R$, and it is possible to express the absorption relation as 


$$
x(a, b, c)=-x(c a \cdot b)=(c a \cdot b) x=-(b, x, c a) .
$$

A nonassociative algebra is called Lie admissible if, under the Lie product $[a, b]=a b-b a$, the algebra is a Lie algebra. This is equivalent to

$$
[[a, b], c]+[[b, c], a]+[[c, a], b]=0 \text {. }
$$

This can also be expressed in terms of associators as

$$
(a, b, c)+(b, c, a)+(c, a, b)-(a, c, b)-(c, b, a)-(b, a, c)=0 .
$$

A $(-1,1)$ algebra is a right alternative Lie admissible algebra. It is defined by the identities

$$
(a, b, c)+(a, c, b)=0, \quad(a, b, c)+(b, c, a)+(c, a, b)=0 .
$$

The absorption formula is given by

$$
\begin{aligned}
x(a, b, c)= & -\frac{1}{3}(x b, c, a)-\frac{1}{3}(x a, c, b)-\frac{1}{3}(x, b, c a)-\frac{1}{3}(x, a, c b) \\
& +\frac{1}{3}(x a, b, c)+\frac{1}{3}(x c, b, a)+\frac{1}{3}(x, a, b c)+\frac{1}{3}(x, c, b a) .
\end{aligned}
$$

Jordan algebras need not be associator ideal algebras. Let $Q$ be the quaternions; then $Q^{+}$is a simple Jordan algebra. $(Q, Q, Q)^{+}$is spanned by $i$, $j$, and $k$; hence $(Q, Q, Q)^{+}$is not an ideal because it is not all of $Q$.

We now give an example of an algebra where $(R, R, R)$ is an ideal, but the algebra is not an associator ideal algebra. A $(-1,1)$ algebra $R$ is called a strongly $(-1,1)$ algebra if $[R,[R, R]]=0$. A strongly $(-1,1)$ algebra is a $(-1,1)$ algebra, and so $(R, R, R)$ is an ideal of $R$. It is immediate that $(R, R$, $R)$ is an ideal of $R^{+}$. In a strongly $(-1,1)$ algebra $(a, b, c)^{+}=2(b, a, c)$; see [9, equation (14)]. Thus $(R, R, R)^{+}=(R, R, R)$ is an ideal of $R^{+}$. We claim that no formula (1) exists for $R^{+}$. We refer to the example of a strongly $(-1$, 1) algebra given in $[4$, Table $I]$.

If an absorption formula (1) holds in $R^{+}$, by the correspondence between associators in $R^{+}$and associators in $R$, the right alternative law and the cyclic law of $R$, we would have an expression holding of the form:

$$
\begin{aligned}
a \circ(b, b, a)^{+}= & \gamma_{1}(a, b, a \circ b)+\gamma_{2}(b, a, a \circ b) \\
& +\gamma_{3}\left(a, a, b^{2}\right)+\gamma_{4}\left(b, b, a^{2}\right) .
\end{aligned}
$$

Using the properties of strongly $(-1,1)$ algebras or $[4$, Table I], we get

$$
2 a(b, b, a)=2 \varepsilon_{1} b(a, a, b)+2 \varepsilon_{2} a(b, b, a)-\left(\varepsilon_{1}+\varepsilon_{2}\right)(a, b,[a, b]),
$$

where $\varepsilon_{1}=\gamma_{3}-\gamma_{1}$ and $\varepsilon_{2}=\gamma_{4}-\gamma_{2}$. This is a dependence relation among the bases and we conclude that $2 \varepsilon_{1}=0,2 \varepsilon_{2}=2, \varepsilon_{1}+\varepsilon_{2}=0$; this is impossible.

Associator dependent algebras. Given a nonassociative algebra $R$ over a field $F, R$ is said to be an associator dependent algebra if it satisfies one or 
more identities of the form:

$$
\begin{aligned}
& \gamma_{1}(a, b, c)+\gamma_{2}(b, c, a)+\gamma_{3}(c, a, b) \\
& \quad+\gamma_{4}(a, c, b)+\gamma_{5}(c, b, a)+\gamma_{6}(b, a, c)=0 .
\end{aligned}
$$

The $\gamma_{1}, \gamma_{2}, \gamma_{3}, \gamma_{4}, \gamma_{5}, \gamma_{6} \in F$ are constants which are not all zero, and the $a, b$, $c$ represent any three elements of $R$. Associator dependent algebras were classified by Kleinfeld et al. in [8]. Right alternative, left alternative, flexible, antiflexible, and $(-1,1)$ algebras are all examples of associator dependent algebras. Less well known associator dependent algebras have also been studied.

Let $H$ be the group ring on $S_{3}$ over $F$ where $F$ is the base field of the nonassociative algebra. If $\pi \in S_{3}$, then the subscript $\pi$ on an associator means that the arguments of the associator are to permuted by $\pi$. We write permutations on the right; the order of multiplication intended for $\pi \sigma$ is shown by $(a, b, c)_{\pi \sigma}=\left[(a, b, c)_{\pi}\right]_{\sigma}$. If $h=\Sigma_{\pi \in S_{3}} \gamma_{\pi} \pi$, then for $a, b, c \in R$, we define

$$
(a, b, c)_{h}=\sum_{\pi \in S_{3}} \gamma_{\pi}(a, b, c)_{\pi} .
$$

Those elements $h$ of $H$ such that $(a, b, c)_{h}=0$ for all elements $a, b, c$ of $R$ are called identities of $R$ (based on the associator). The set of all these identities of $R$ form a left ideal of $H$.

The study of the identities of type (4) is equivalent to the study of different left ideals of $H$. If we assume that $F$ is not of characteristic 2 or 3, we may write $H=H_{1} \oplus H_{2} \oplus H_{3}$, where $H_{1}$ and $H_{2}$ are $1 \times 1$ matrices and $H_{3}$ is $2 \times 2$.

The following representation is used for $S_{3}$ in this paper.

$$
\begin{aligned}
(12) & =(1) \oplus(-1) \oplus\left(\begin{array}{cc}
1 & 0 \\
-1 & -1
\end{array}\right), \\
(123) & =(1) \oplus(1) \oplus\left(\begin{array}{cc}
-1 & -1 \\
1 & 0
\end{array}\right) .
\end{aligned}
$$

If $L$ is a left ideal of $H$, then $L=L_{1} \oplus L_{2} \oplus L_{3}$. Since $H_{1}$ and $H_{2}$ are one-dimensional vector spaces, their only left ideals are 0 and the whole space. There are three types of left ideals of $H_{3}$. They are $0, H_{3}$, or a left ideal generated by a matrix of the form $\left(\begin{array}{ll}\alpha & \beta \\ 0 & 0\end{array}\right)$ where $\alpha$ and $\beta$ are not both zero. We shall simply write $\left(\begin{array}{cc}\alpha & \beta \\ 0 & 0\end{array}\right)$ to represent the left ideal of $H_{3}$ generated by $\left(\begin{array}{ll}\alpha & \beta \\ 0 & 0\end{array}\right)$. The left ideal generated by $\left(\begin{array}{ll}\alpha & \beta \\ 0 & 0\end{array}\right)$ corresponds to an identity of this form:

$$
\begin{aligned}
\alpha((a, b, c)+ & (b, a, c)-(b, c, a)-(c, b, a)) \\
+ & \beta((a, c, b)-(c, a, b)+(b, c, a)-(b, a, c))=0 .
\end{aligned}
$$


We now list a few well-known structures.

$H_{1}$ corresponds to third power associativity: $a^{2} a=a a^{2}$.

$\mathrm{H}_{2}$ corresponds to Lie admissibility.

$H_{1} \oplus H_{2}$ corresponds to the cyclic law: $(a, b, c)+(b, c, a)+(c, a, b)=0$.

$H_{1} \oplus H_{3}$ corresponds to the alternative law: $(a, b, c)=(\operatorname{sgn} \pi)(a, b, c)_{\pi \cdot}$.

$H_{1} \oplus\left(\begin{array}{lll}1 & 1 \\ 0 & 0\end{array}\right)$ corresponds to the right alternative law: $(a, b, c)+(a, c, b)=0$.

$H_{1} \oplus H_{2} \oplus\left(\begin{array}{ll}1 & 1 \\ 0 & 0\end{array}\right)$ corresponds to $(-1,1)$ algebras.

An algebra is considered a maximal associator dependent algebra if its left ideal of identities is a maximal left ideal. One can see that alternative algebras are certainly maximal. The type described by $\mathrm{H}_{2} \oplus \mathrm{H}_{3}$ is also maximal; this type is not power associative. They satisfy $(a, b, c)=(a, b, c)$, for all $\pi \in S_{3}$.

The third type of maximal associator dependent algebras is defined by

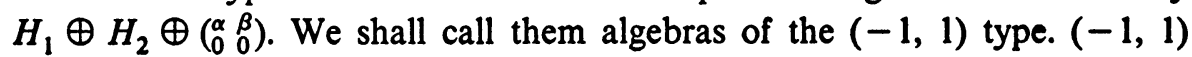
algebras, flexible Lie admissible algebras, and antiflexible third power associative algebras are all of this type.

Various types of maximal associator dependent algebras have been studied, and many results can be found in the literature.

THEOREM 1. If $R$ is an associator ideal algebra with an identity element, then $R$ is Lie admissible.

Proof. We remark that we do not say that $R$ itself is a Lie algebra. We say that $R^{-}$, i.e. $R$ with the product $[a, b]=a b-b a$, is a Lie algebra. We assume $R$ satisfies an identity of the type (1).

Consider $\boldsymbol{\Sigma}_{\boldsymbol{\sigma} \in S_{4}}(\operatorname{sgn} \sigma) a(b, c, d)_{\boldsymbol{\sigma}}$; let $d=1$. This sum reduces to

$$
\begin{array}{r}
-(a, b, c)-(b, c, a)-(c, a, b)+(a, c, b)+(c, b, a)+(b, a, c) \\
=-\sum_{\tau \in S_{3}}(\operatorname{sgn} \tau)(a, b, c)_{\tau} .
\end{array}
$$

If this alternating sum can be shown to be zero, $R$ will be Lie admissible. If we apply (1) to each of the 24 terms in $\Sigma_{\sigma \in S_{4}}(\operatorname{sgn} \sigma) a(b, c, d)_{\sigma}$, we have a sum of $24 \cdot 3 \cdot 24$ terms. Fix $\pi \in S_{4}$. The terms involving $\lambda_{\pi}$ when collected become $\pm \lambda_{\pi} \Sigma_{\sigma \in S_{4}}(\operatorname{sgn} \sigma)(a b, c, d)_{\sigma}$. Since $d=1$, the only nonzero associators will have $d$ as a factor of the argument in the left entry of the associator. Each of the associators involving $a, b, c$ will occur twice in the sum because $d$ will occur as the left factor as well as the right factor of the product entry. Since these two arrangements will differ by a transposition, they will have opposite signs and will cancel. Similarly, all $\mu_{\pi}$ and $\nu_{\pi}$ terms cancel. This shows that $\Sigma_{\tau \in S_{3}}(\operatorname{sgn} \tau)(a, b, c)_{\tau}$ is an identity of $R$, and thus $R$ is Lie admissible.

COROLlary. A right alternative associator ideal algebra with an identity element is a $(-1,1)$ algebra. 
Proof. A $(-1,1)$ algebra is a right alternative Lie admissible algebra.

COROLlARY. If $R$ is an alternative associator ideal algebra with an identity element, then $R$ is associative.

Proof. Since $R$ is Lie admissible, $0=\Sigma_{\tau \in S_{3}}(\operatorname{sgn} \tau)(a, b, c)_{\tau}=6(a, b, c)$.

THEOREM 2. Except for the cases $\left(\begin{array}{ll}0 & 1 \\ 0 & 1\end{array}\right)$ and $\left(\begin{array}{ll}2 & 1 \\ 0 & 0\end{array}\right)$, a nonassociative algebra of the $(-1,1)$ type is an associator ideal algebra.

Proof. Let $K$ be the group ring on $S_{4}$ over $F$. If $k=\Sigma_{\pi \in S_{4}} \gamma_{\pi} \pi$, associate with $k$ the formula $\Sigma_{\pi \in S_{4}} \gamma_{\pi} a(b, c, d)_{\pi}$. We let $(R) k$ represent the set of elements produced by the formula of $k$ as the four arguments vary throughout $R$. $(R, R, R)$ may be only an additive group, but the set $N=\left\{k \in K \mid(R) k \subseteq_{f}(R, R, R)\right\}$ will be a left ideal of the group ring $K$.

We will now show that each associator dependence relation on $R$ generates two elements of $N$. If $\Sigma_{\tau \in S_{3}} \gamma_{\tau}(a, b, c)_{\tau}=0$ is an associator dependence relation for $R$, then we have that

$$
d \sum_{\tau \in S_{3}} \gamma_{\tau}(a, b, c)_{\tau}=0 \text { and }\left[\sum_{\tau \in S_{3}} \gamma_{\tau}(a, b, c)_{\tau}\right] d=0
$$

are identities as well. This last identity can be rewritten using (2) as

$$
\sum_{\tau \in S_{3}} \gamma_{\tau} a(b, c, d)_{\tau} \subseteq_{f}(R, R, R)
$$

where $\tau$ permutes the first three positions; the fourth position is left fixed.

These are the two elements of $N$. In our case, the cyclic law and the $\left(\begin{array}{ll}\alpha & \beta \\ 0 & 0\end{array}\right)$ law hold for $R$; they give us these four elements of $N$.

$$
\begin{aligned}
k_{1}= & (1234)+(14)+(1324), \\
k_{2}= & I+(123)+(132), \\
k_{3}= & \alpha((1234)+(134)-(14)-(14)(23)) \\
& +\beta((124)-(1324)+(14)-(134)), \\
k_{4}= & \alpha(I+(12)-(132)-(13))+\beta((23)-(123)+(132)-(12)) .
\end{aligned}
$$

As $F$ is not of characteristic 2 or $3, K$ is isomorphic to $F_{1 \times 1} \oplus F_{1 \times 1} \oplus F_{2 \times 2}$ $\oplus F_{3 \times 3} \oplus F_{3 \times 3}[1$, p. 108].

Knowing the images of the generators of $S_{4},(12)$ and (234) enables one to compute the entire representation. We include in the listing the other needed elements as well. 


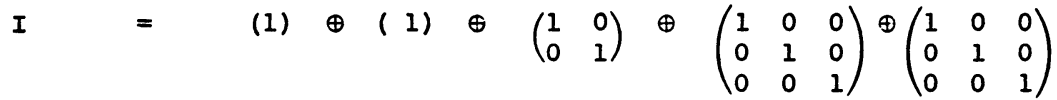

$$
\begin{aligned}
& \text { (12) }=\quad(1) \oplus(-1) \oplus\left(\begin{array}{rr}
1 & 0 \\
-1 & -1
\end{array}\right) \oplus\left(\begin{array}{rrr}
-1 & 0 & 0 \\
-1 & 1 & 0 \\
-1 & 0 & 1
\end{array}\right) \oplus\left(\begin{array}{rrr}
1 & 0 & 0 \\
1 & -1 & 0 \\
1 & 0 & -1
\end{array}\right) \\
& \text { (13) }=\quad(1) \oplus(-1) \oplus\left(\begin{array}{rr}
-1 & -1 \\
0 & 1
\end{array}\right) \oplus\left(\begin{array}{lll}
1 & -1 & 0 \\
0 & -1 & 0 \\
0 & -1 & 1
\end{array}\right) \oplus\left(\begin{array}{rrr}
-1 & 1 & 0 \\
0 & 1 & 0 \\
0 & 1 & -1
\end{array}\right) \\
& (14)=(1) \oplus(-1) \oplus\left(\begin{array}{ll}
0 & 1 \\
1 & 0
\end{array}\right) \oplus\left(\begin{array}{lll}
1 & 0 & -1 \\
0 & 1 & -1 \\
0 & 0 & -1
\end{array}\right) \oplus\left(\begin{array}{rrr}
-1 & 0 & 1 \\
0 & -1 & 1 \\
0 & 0 & 1
\end{array}\right) \\
& \text { (23) }=(1) \oplus(-1) \oplus\left(\begin{array}{ll}
0 & 1 \\
1 & 0
\end{array}\right) \oplus\left(\begin{array}{lll}
0 & 1 & 0 \\
1 & 0 & 0 \\
0 & 0 & 1
\end{array}\right) \oplus\left(\begin{array}{rrr}
0 & -1 & 0 \\
-1 & 0 & 0 \\
0 & 0 & -1
\end{array}\right)
\end{aligned}
$$

The elements $k_{1}, k_{2}, k_{3}, k_{4}$ have these representations in the group algebra.

$$
\begin{aligned}
& \text { (123) }=\quad(1) \oplus(1) \oplus\left(\begin{array}{rr}
-1 & -1 \\
1 & 0
\end{array}\right) \oplus\left(\begin{array}{lll}
-1 & 1 & 0 \\
-1 & 0 & 0 \\
-1 & 0 & 1
\end{array}\right) \oplus\left(\begin{array}{lll}
-1 & 1 & 0 \\
-1 & 0 & 0 \\
-1 & 0 & 1
\end{array}\right)
\end{aligned}
$$

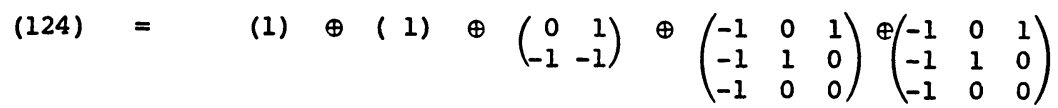

$$
\begin{aligned}
& (132)=(1) \oplus(1) \oplus\left(\begin{array}{rr}
0 & 1 \\
-1 & -1
\end{array}\right) \oplus\left(\begin{array}{lll}
0 & -1 & 0 \\
1 & -1 & 0 \\
0 & -1 & 1
\end{array}\right) \oplus\left(\begin{array}{lll}
0 & -1 & 0 \\
1 & -1 & 0 \\
0 & -1 & 1
\end{array}\right) \\
& (134)=(1) \oplus(1) \oplus\left(\begin{array}{rr}
-1 & -1 \\
1 & 0
\end{array}\right) \oplus\left(\begin{array}{lll}
1 & -1 & 0 \\
0 & -1 & 1 \\
0 & -1 & 0
\end{array}\right) \oplus\left(\begin{array}{lll}
1 & -1 & 0 \\
0 & -1 & 1 \\
0 & -1 & 0
\end{array}\right) \\
& (234)=(1) \oplus(1) \oplus\left(\begin{array}{rr}
0 & 1 \\
-1 & -1
\end{array}\right) \oplus\left(\begin{array}{lll}
0 & 1 & 0 \\
0 & 0 & 1 \\
1 & 0 & 0
\end{array}\right) \oplus\left(\begin{array}{lll}
0 & 1 & 0 \\
0 & 0 & 1 \\
1 & 0 & 0
\end{array}\right) \\
& (1234)=\quad(1) \oplus(-1) \oplus\left(\begin{array}{rr}
-1 & -1 \\
0 & 1
\end{array}\right) \oplus\left(\begin{array}{lll}
-1 & 1 & 0 \\
-1 & 0 & 1 \\
-1 & 0 & 0
\end{array}\right) \oplus\left(\begin{array}{rrr}
1 & -1 & 0 \\
1 & 0 & -1 \\
1 & 0 & 0
\end{array}\right) \\
& (1324)=\quad(1) \oplus(-1) \oplus\left(\begin{array}{rr}
1 & 0 \\
-1 & -1
\end{array}\right) \oplus\left(\begin{array}{lll}
0 & -1 & 1 \\
1 & -1 & 0 \\
0 & -1 & 0
\end{array}\right) \oplus\left(\begin{array}{rrr}
0 & 1 & -1 \\
-1 & 1 & 0 \\
0 & 1 & 0
\end{array}\right) \\
& \text { (14) }(23)=
\end{aligned}
$$

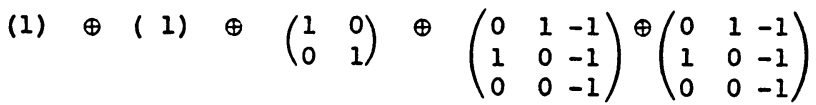


By inspection, one sees that any left ideal containing $k_{1}$ contains both $1 \times 1$ summands. Similarly, any left ideal containing $k_{1}, k_{2}, k_{3}$, and $k_{4}$ will contain both $3 \times 3$ summands no matter what values of $(\alpha, \beta) \neq(0,0)$ are chosen. Any left ideal containing $k_{3}$ and $k_{4}$ will contain the $2 \times 2$ summand unless $\left(\begin{array}{ll}\alpha & \beta \\ 0 & 0\end{array}\right)$ are of type $\left(\begin{array}{ll}0 & 1 \\ 0 & 0\end{array}\right)$ or $\left(\begin{array}{ll}2 & 1 \\ 0 & 0\end{array}\right)$. Except for the two exceptional cases, the left ideal of identities of $R(R, R, R) \bmod (R, R, R)$ contains all of $K$. We have shown $(R) I \subseteq(R, R, R)$. This means $a(b, c, d) \subseteq(R, R, R)$ for all $a, b$, $c, d \in R$, and consequently $(R, R, R)$ is an ideal of $R$.

The exceptional case $\left(\begin{array}{ll}0 & 1 \\ 0 & 0\end{array}\right)$ corresponds to flexible, Lie admissible algebras. Jordan algebras are of such a type; $Q^{+}$, where $Q$ is the quaternions, shows the associators need not be an ideal. The case $\left(\begin{array}{ll}2 & 1 \\ 0 & 0\end{array}\right)$ is the antiflexible, third power associative algebras. These algebras are specified by the identities $(a, b$, $c)+(b, c, a)+(c, a, b)=0$ and $(a, b, c)=(c, b, a)$. The structure of these algebras has been studied in [2], but it is not known if in these rings, the additive span of the associators is an ideal.

Alternator ideal algebras. We now will briefly discuss alternator ideal algebras. An alternator is an associator of the form $(a, a, b),(a, b, a)$, or $(b, a$, $a)$; i.e. two entries are identical. An alternator ideal algebra is one where the additive subgroup spanned by the collection of all alternators is an ideal. As usual, we insist that there be a formula which expresses the absorption. In the associator ideal case, it was sufficient to find a formula for one-sided absorption. For alternators, one needs a formula with six parts. We need to be able to absorb elements on the left side and on the right side for each of three types of alternators. The simplest examples of alternator ideal algebras are the alternative algebras, since all alternators of alternative algebras are zero. The next theorem shows that algebras of type $(-1,1)$ form another class of such algebras.

THEOREM 3. Any associator ideal algebra with an identity element is an alternator ideal algebra.

Proof. An associator ideal algebra with an identity element is Lie admissible by Theorem 1. Thus $0=\Sigma_{\tau \in S_{3}}(\operatorname{sgn} \tau)(a, b, c)_{\tau} \equiv 6(a, b, c)$ modulo alternators. This says that the space spanned by the associators is the same as the space spanned by the alternators. Since the span of the associators is an ideal, the span of the alternators is the same ideal.

Right alternative alternator ideal algebras. Let us define $M(a, b, c)=(a, b$, $c)+(b, a, c)$. In any algebra satisfying $(x, x, x)=0$ :

$$
\begin{gathered}
M(a, b, c)=M(b, a, c), \\
M(a, b, c)+M(b, c, a)+M(c, a, b)=0 .
\end{gathered}
$$


We will examine right alternative algebras satisfying an identity of the following type.

$$
\begin{aligned}
{[a,(b, b, c)]=} & \alpha_{1} M([a, b], b, c)+\alpha_{2} M([a, b], c, b) \\
& +\alpha_{3} M([a, c], b, b) \\
& +\alpha_{4} M([b, c], a, b)+\alpha_{5} M([b, c], b, a) .
\end{aligned}
$$

By virtue of (5),

$$
\begin{array}{r}
M([R, R], R, R)+M(R,[R, R], R)+M(R, R,[R, R]) \\
\subseteq_{f} M([R, R], R, R) .
\end{array}
$$

We are thus considering all right alternative algebras where a formula exists such that

$$
\begin{array}{rl}
{[R, M(R, R, R)] \subseteq_{f}} & M([R, R], R, R) \\
& +M(R,[R, R], R)+M(R, R,[R, R]) .
\end{array}
$$

THEOREM 4 (THEDY). Let $R$ be a right alternative algebra. $R$ is an alternator ideal algebra $\Leftrightarrow[R, M(R, R, R)] \subseteq_{f} M(R, R, R)$.

Proof. See [9, Corollary to Lemma 11, p. 23].

COROLlARY. If $R$ is a right alternative algebra satisfying (6), then $R$ is an alternator ideal algebra.

We shall refer to specific algebras satisfying (6) by listing their coefficients as $\left(\alpha_{1}, \alpha_{2}, \alpha_{3}, \alpha_{4}, \alpha_{5}\right)$.

The algebra $(0,0,0,0,0)$ was given in [9, Theorem 4]. It is clear that [ $R$, $M(R, R, R)]=0$, and, as Thedy showed, $M(R, R, R)$ is an ideal whose square is zero. It follows that semiprime algebras of type $(0,0,0,0,0)$ are alternative. We shall not examine this case any further, and we shall now assume that in any such formula under discussion, at least one coefficient is not zero.

The algebra $(0,0,0,0,1)$ was given in [3] and [5, Equation (5)]. It is a right alternative algebra satisfying $\left(a, b^{2}, c\right)=b \circ(a, b, c)$. It is shown in [5] that in such algebras, $M(R, R, R)$ is an ideal whose square is zero.

A $(-1,1)$ algebra is of the type $\left(\frac{1}{4},-\frac{1}{4}, 0,-\frac{1}{4}, \frac{1}{4}\right)$.

A strongly $(-1,1)$ algebra is a $(-1,1)$ algebra, so it will satisfy $\left(\frac{1}{4},-\frac{1}{4}, 0\right.$, $\left.-\frac{1}{4}, \frac{1}{4}\right)$. Since in a strongly $(-1,1)$ algebra $(x, x,[x, a])=0$, the five terms on the right-hand side of (6) are dependent. That is:

$$
M([a, b], b, c)+M([a, b], c, b)+M([a, c], b, b)=0
$$

and

$$
M([b, c], a, b)+M([b, c], b, a)+M([a, c], b, b)=0 .
$$


Thus strongly $(-1,1)$ algebras will satisfy (6) for many choices of coefficients including $\left(0,-\frac{1}{2},-\frac{1}{2},-\frac{1}{2}, 0\right)$.

If $R$ is a right alternative alternator ideal algebra, then by hypothesis,

$$
[R, M(R, R, R)] \subseteq_{f} M(R, R, R) .
$$

This is not (6), because in (6) we assume that

$$
\begin{aligned}
{[R, M(R, R, R)] \subseteq_{f} M([R, R], R, R)+M(R,[R, R], R) } \\
\\
+M(R, R,[R, R]) \\
\subseteq_{f} M([R, R], R, R) .
\end{aligned}
$$

We now ask under what conditions a right alternative alternator ideal algebra will satisfy (6). If (6) is satisfied, then $[a,(a, a, c)]=\gamma(a, a,[a, c])$ where $\gamma=-\alpha_{3}-\alpha_{4}-\alpha_{5}$. Such an identity is common. This identity holds in any flexible algebra for $\gamma=1$, and in any algebra of the $(-1,1)$ type except flexible Lie admissible for $\gamma=0$.

THEOREM 5. Let $R$ be a right alternative alternator ideal algebra with an identity element. $R$ satisfies $(6) \Leftrightarrow$ for some $\gamma \in F$,

$$
[a,(a, a, b)]=\gamma(a, a,[a, b])
$$

is an identity of $R$.

Proof. We have already shown that (6) implies

$$
[a,(a, a, b)]=\left(-\alpha_{3}-\alpha_{4}-\alpha_{5}\right)(a, a,[a, b]) .
$$

The assumption of alternator ideal and (5) says that

$$
[a, M(b, c, d)]=\sum_{\pi \in S_{4}} \gamma_{\pi} M(a b, c, d)_{\pi} .
$$

We can rewrite this as

$$
\begin{aligned}
{[a,(b, b, c)]=} & \delta_{1} M(a b, b, c) \\
& +\delta_{2} M(a b, c, b)+\delta_{3} M(a c, b, b) \\
& +\delta_{4} M(b c, a, b)+\delta_{5} M(b c, b, a)+\delta_{6} M\left(b^{2}, a, c\right) \\
& +\delta_{7} M\left(b^{2}, c, a\right)+M([R, R], R, R)
\end{aligned}
$$

where the $+M([R, R], R, R)$ is expressed by some formula. In any right alternative algebra by (2) and (3) we have $M\left(x^{2}, y, x\right)-2 M(x y, x, x)=$ $-[x,(x, x, y)]+(x, x,[x, y])$. The right-hand side of this identity lies in $M([R, R], R, R)$ by assumption. If we linearize the left-hand side in three ways we get the following: 


$$
\left.\begin{array}{l}
M\left(b^{2}, a, c\right)-2 M(b a, b, c) \\
\quad+M(b c, a, b)-2 M(b a, c, b) \\
\quad+M(c b, a, b)-2 M(c a, b, b)
\end{array}\right\} \in M([R, R], R, R)
$$

These three dependence relations allow us to reduce the number of elements of the spanning set and to say that

$$
\begin{aligned}
{[a,(b, b, c)]=} & \varepsilon_{1} M(a b, b, c)+\varepsilon_{2} M(a b, c, b) \\
& +\varepsilon_{3} M(a c, b, b)+\varepsilon_{4} M(b c, a, b)+M([R, R], R, R) .
\end{aligned}
$$

If we linearize this by first replacing $a$ by 1 , then $c$ by 1 , and finally $b$ by $b+1$, we get

$$
\begin{aligned}
& 0=\left(2 \varepsilon_{1}-\varepsilon_{2}-\varepsilon_{3}\right)(b, b, c), \\
& 0=-\left(\varepsilon_{3}+\varepsilon_{4}\right)(b, b, a), \\
& 0=\varepsilon_{1} M(a, b, c)+\left(\varepsilon_{2}+\varepsilon_{4}\right) M(c, a, b) .
\end{aligned}
$$

The last equation is an associator dependent equation. Since its representation in $R_{2 \times 2}$ is

$$
\left(\begin{array}{cc}
2 \varepsilon_{1}-\left(\varepsilon_{2}+\varepsilon_{4}\right) & 0 \\
-\varepsilon_{1}+2\left(\varepsilon_{2}+\varepsilon_{4}\right) & 0
\end{array}\right),
$$

it will imply $R$ is alternative unless $\varepsilon_{1}=\varepsilon_{2}+\varepsilon_{4}=0$.

If $R$ is not alternative, then $0=2 \varepsilon_{1}-\varepsilon_{2}-\varepsilon_{3}=\varepsilon_{3}+\varepsilon_{4}=\varepsilon_{1}=\varepsilon_{2}+\varepsilon_{4}$; these give $\varepsilon_{1}=\varepsilon_{2}=\varepsilon_{3}=\varepsilon_{4}=0$. We have shown that

$$
[a,(b, b, c)] \in M([R, R], R, R)
$$

by some formula.

Suppose that $R$ is a right alternative ring satisfying an identity of form (6) which contains an identity element 1 and an idempotent $e \neq 0, \neq 1$ such that $(e, e, R)=0$. We will show that with two possible exceptions, if $R$ is prime, then $R$ is alternative. 
From the right alternative property and the assumption on the idempotent, we can write $R$ as the additive direct sum of the four summands $R=R_{11}+$ $R_{10}+R_{01}+R_{00}$ where $x \in R_{i j} \Leftrightarrow e x=i x$ and $x e=j x$. This "Peirce" decomposition has a multiplication between the summands which is given below; see [6, Lemma 6, p. 166]. We write $R_{1}$ for $R_{11}$ and $R_{0}$ for $R_{00}$.

\section{TABLE I}

$\begin{array}{lllll} & R_{1} & R_{10} & R_{01} & R_{0} \\ R_{1} & R_{1}+R_{01} & R_{10} & R_{10} & 0 \\ R_{10} & 0 & R_{1}+R_{01} & R_{1} & R_{10} \\ R_{01} & R_{01} & R_{0} & R_{0}+R_{10} & 0 \\ R_{0} & 0 & R_{01} & R_{01} & R_{10}+R_{0}\end{array}$

Furthermore, $x_{10}^{2} \in R_{1}$ and $x_{01}^{2} \in R_{0} . R_{1}^{+}$and $R_{0}^{+}$are Jordan algebras.

We shall not refer to this table specifically in this paper, but it will be used repeatedly in all that we do.

Table $I$ is said to be the table of a right alternative algebra $R$ with an idempotent $e \neq 0, \neq 1$ satisfying $(e, e, R)=0$. If the products between summands behave as $R_{i j} R_{k l} \subseteq \delta_{j k} R_{i l}$ except that $R_{10} R_{10} \subseteq R_{01}$ and $R_{01} R_{01} \subseteq$ $R_{10}\left(\delta_{i i}=1, \delta_{i j}=0\right.$ if $\left.i \neq j\right)$, we shall say that $R$ has an alternative table. In an alternative table, the summands multiply as matrix units, the exceptions being $R_{10} R_{10} \subseteq R_{01}$ and $R_{01} R_{01} \subseteq R_{10}$.

With the possible exception of two cases, these two statements hold. If $R$ is semiprime, then $R$ has an alternative table. If $R$ is prime, then $R$ is alternative.

We shall use the subscript notation to specify a particular component of a product we wish to examine. Thus $\left(x_{10} y_{10}\right)_{1}$ means the summand of the product $x_{10} y_{10}$ which is in $R_{1}$. From the table we see, for example, that $\left(x_{10} y_{10}\right)_{10}=0$. Using the table, we often write equalities of the form $\left[\left(x_{10} y_{10}\right) z_{10}\right]_{10}=\left(x_{10} y_{10}\right) z_{10} z_{10}$.

Since our algebra has an identity element, the idempotent $e^{\prime}=1-e$ satisfies $e^{\prime} \neq 0, \neq 1$ and $\left(e^{\prime}, e^{\prime}, R\right)=0$. The decomposition of $R$ with respect to $e^{\prime}$ is exactly the same except that the subscripts are interchanged. Thus, if we can show that $R_{10} R_{10} \subseteq R_{01}$, by "reversing subscripts" it immediately follows that $R_{01} R_{01} \subseteq R_{10}$.

In all our proofs, the technique is based on the fact that the identity (6) holds for all elements in the algebra. We compute the expression for all possible arrangements of $a, b, c, d$ in $[a,(b, c, d)+(c, b, d)]$. We then compare the various equations we get and decide if any $\left(\alpha_{1}, \alpha_{2}, \alpha_{3}, \alpha_{4}, \alpha_{5}\right)$ can possibly satisfy the requirements.

LeMma 1. $R_{1} R_{1} \subseteq R_{1}$ and $R_{0} R_{0} \subseteq R_{0}$. 
Proof. Since $a_{1} b_{1} \subseteq R_{1}+R_{01}$, it suffices to show that $\left(a_{1} b_{1}\right)_{01}=0$. We know $\left[e,\left(e, a_{1}, b_{1}\right)+\left(a_{1}, e, b_{1}\right)\right]=\left[e,\left(a_{1} b_{1}\right)_{01}\right]=-\left(a_{1} b_{1}\right)_{01}$. From (6) we have

$$
\left[e,\left(e, a_{1}, b_{1}\right)+\left(a_{1}, e, b_{1}\right)\right] \in M\left(\left[e, R_{1}\right], R, R\right)+M([R, R], e, e)=0 .
$$

We have shown $\left(R_{1} R_{1}\right)_{01}=0$. The second statement is obtained by reversing subscripts.

Practically every statement we make will be false for some particular choice of coefficients $\left(\alpha_{1}, \alpha_{2}, \alpha_{3}, \alpha_{4}, \alpha_{5}\right)$. To make the theorems readable, we adopt the following form. We state the important result of the theorem in the first sentence. The second sentence gives the exceptions.

We will need to know more about the product $R_{10} R_{10} \subseteq R_{1}+R_{01}$. In particular, when does the stronger statement $R_{10} R_{10} \subseteq R_{01}$ hold? Suppose $R_{10} R_{10} \& R_{01}$. Then there exists an $x_{10}$ and a $y_{10}$ such that $x_{10} y_{10}=a_{1}+a_{01}$ where $a_{1} \neq 0$. Let $y_{10} x_{10}=b_{1}-a_{01}$. We use (6) to give us these relations.

$\begin{array}{lccc} & M\left(x_{10}, y_{10}, e\right) & M\left(e, x_{10}, y_{10}\right) & M\left(e, y_{10}, x_{10}\right) \\ \text { 1. }\left[e, M\left(x_{10}, y_{10}, e\right)\right]= & 2 \alpha_{1}-2 \alpha_{5} & \alpha_{2}-\alpha_{4} & \alpha_{2}-\alpha_{4} \\ \text { 2. }\left[e, M\left(e, x_{10}, y_{10}\right)\right]= & \alpha_{2}+\alpha_{3}+\alpha_{5} & \alpha_{1} & \alpha_{3}+\alpha_{4} \\ \text { 3. }\left[x_{10},\left(e, e, y_{10}\right)\right]= & -\alpha_{2}+\alpha_{4} & -\alpha_{1} & \alpha_{5} \\ \text { 4. }\left[x_{10}, M\left(e, y_{10}, e\right)\right]= & -\alpha_{1}-\alpha_{3}-\alpha_{4} & -\alpha_{2}-\alpha_{3} & -\alpha_{5}\end{array}$

$M\left(x_{10}, y_{10}, e\right)=a_{1}+b_{1}, M\left(e, x_{10}, y_{10}\right)=-a_{1}, M\left(e, y_{10}, x_{10}\right)=-b_{1}$. These imply that the left-hand side of each of the four equations listed above is zero.

Interchanging $x_{10}$ and $y_{10}$ will give the same equations with $a_{1}$ and $b_{1}$ interchanged. If we subtract each equation from the one gotten by interchanging $x_{10}$ and $y_{10}$, we get $\left(\alpha_{1}-\alpha_{3}-\alpha_{4}\right)\left(a_{1}-b_{1}\right)=0,\left(\alpha_{1}+\alpha_{5}\right)\left(a_{1}-b_{1}\right)$ $=0$ and $\left(\alpha_{2}+\alpha_{3}-\alpha_{5}\right)\left(a_{1}-b_{1}\right)=0$. If $a_{1} \neq b_{1}$, then these three coefficients must be 0 . Solving them simultaneously implies the algebra is of the type $(\alpha,-\alpha-\beta, \beta, \alpha-\beta,-\alpha)$. If we substitute these expressions into the equation, we get $\alpha\left(a_{1}+b_{1}\right)=0$. This means that if $a_{1} \neq \pm b_{1}$, the algebra must further be restricted to the type $(0,-\beta, \beta,-\beta, 0)$. If $a_{1}=b_{1}$, since we assumed $a_{1} \neq 0$, the coefficients must satisfy these four equations.

$\begin{array}{cccccc}4 \alpha_{1} & -2 \alpha_{2} & & +2 \alpha_{4} & -4 \alpha_{5} & =0 \\ -\alpha_{1} & +2 \alpha_{2} & +\alpha_{3} & -\alpha_{4} & +2 \alpha_{5} & =0 \\ \alpha_{1} & -2 \alpha_{2} & & +2 \alpha_{4} & -\alpha_{5} & =0 \\ -2 \alpha_{1} & +\alpha_{2} & -\alpha_{3} & -2 \alpha_{4} & +\alpha_{5} & =0\end{array}$

Solving these simultaneously gives the solution $(\alpha, \beta,-\alpha-\beta, \beta, \alpha)$. By the above proof and by reversing subscripts, we have shown the following lemma.

LEMMA 2. $R_{10} R_{10} \subseteq R_{01}$ and $R_{01} R_{01} \subseteq R_{10}$. The only exceptions are the cases $(\alpha,-\alpha-\beta, \beta, \alpha-\beta,-\alpha)$ and $(\alpha, \beta,-\alpha-\beta, \beta, \alpha)$. 
We shall now assume that $R_{1} R_{01}+R_{0} R_{10} \neq 0$. We will show that if $R$ is semiprime, then $R_{1} R_{01}+R_{0} R_{10}=0$.

LEMMA 3. If $R_{1} R_{01}+R_{0} R_{10} \neq 0$, then $R$ is of the type $(-\alpha, 0, \alpha, 0,1-\alpha)$.

Proof. We may assume that $x_{1} y_{01} \neq 0$ for some choice of $x_{1} \in R_{1}$ and $y_{01} \in R_{01}$. The other case where $R_{1} R_{01}=0$ but $R_{0} R_{10} \neq 0$ is done by reversing subscripts.

We examine these equations:

$$
\begin{array}{lcc} 
& M\left(x_{1}, y_{01}, e\right) & M\left(e, y_{01}, x_{1}\right) \\
{\left[e, M\left(e, x_{1}, y_{01}\right)\right]=} & -\alpha_{3}-\alpha_{5} & -\alpha_{3}-\alpha_{4} \\
{\left[e, M\left(e, y_{01}, x_{1}\right)\right]=} & -\alpha_{2} & -\alpha_{1} \\
{\left[e, M\left(x_{1}, y_{01}, e\right)\right]=} & -\alpha_{1}+\alpha_{5} & -\alpha_{2}+\alpha_{4} \\
{\left[x_{1},\left(e, e, y_{01}\right)\right]=} & -\alpha_{4} . & -\alpha_{5}
\end{array}
$$

Expanding the alternators gives $M\left(e, x_{1}, y_{01}\right)=x_{1} y_{01}=-M\left(x_{1}, y_{01}, e\right)$, and $M\left(e, y_{01}, x_{1}\right)=0$.

We get these equations: $\alpha_{3}+\alpha_{5}=1, \alpha_{2}=0, \alpha_{1}-\alpha_{5}=-1, \alpha_{4}=0$. The solution to this system is $(-\alpha, 0, \alpha, 0,1-\alpha)$.

THEOREM 6. Let $R$ be a right alternative algebra satisfying an identity of form (6). If $R$ has an identity element 1 and an idempotent $e \neq 0, \neq 1$ such that $(e, e$, $R)=0$, then $R_{1} R_{01}+R_{0} R_{10}$ is a trivial ideal of $R$. The case $(0,0,0,0,1)$ is excluded.

Proof. We will show $\left(R_{1} R_{01}\right) R+R\left(R_{1} R_{01}\right) \subseteq R_{1} R_{01}+R_{0} R_{10}$. The products involving $R_{0} R_{10}$ are proved by reversing subscripts. We first notice that, by Lemma 2 and Lemma 3, $R_{10} R_{10} \subseteq R_{01}$ and $R_{01} R_{01} \subseteq R_{10}$. We now consider the eight cases separately. 1. $\left(R_{1} R_{01}\right) R_{1} \subseteq R_{10} R_{1}=0.2 .\left(R_{1} R_{01}\right) R_{10}$ $\subseteq\left(R_{1}, R_{10}, R_{01}\right) \subseteq R_{1}$ while $\left(R_{1} R_{01}\right) R_{10} \subseteq R_{10}^{2} \subseteq R_{01}$. Thus $\left(R_{1} R_{01}\right) R_{10}=0$. 3. $\left(R_{1} R_{01}\right) R_{01}$ is the most difficult and is done last. 4. $\left(R_{1} R_{01}\right) R_{0} \subseteq\left(R_{1}, R_{0}\right.$, $\left.R_{01}\right) \subseteq R_{1} R_{01}$. 5. $R_{1}\left(R_{1} R_{01}\right) \subseteq\left(R_{1} R_{1}\right) R_{01}+\left(R_{1}, R_{01}, R_{1}\right) \subseteq R_{1} R_{01}$. 6. $R_{10}\left(R_{1} R_{01}\right) \subseteq\left(R_{10}\right)^{2} \subseteq R_{01} \cdot R_{10}\left(R_{1} R_{01}\right) \subseteq\left(R_{10}, R_{01}, R_{1}\right) \subseteq R_{1}$. Thus $R_{10}\left(R_{1} R_{01}\right)=0^{*}$. 7. $R_{01}\left(R_{1} R_{01}\right) \subseteq R_{0}$ and yet $R_{01}\left(R_{1} R_{01}\right) \subseteq\left(R_{01} R_{1}\right) R_{01}+$ $\left(R_{01}, R_{01}, R_{1}\right) \subseteq R_{10}$. Thus $R_{01}\left(R_{1} R_{01}\right)=0 * *$. 8. $R_{0}\left(R_{1} R_{01}\right) \subseteq R_{0} R_{10}$. We shall now prove the omitted case, item 3. $\left[r_{01}, M\left(e, x_{1}, x_{01}\right)\right]=\left[r_{01}, x_{1} x_{01}\right]=$ $r_{01}\left(x_{1} x_{01}\right)-\left(x_{1} x_{01}\right) r_{01}=-\left(x_{1} x_{01}\right) r_{01}$ by ${ }^{* *}$. We have shown

$$
\left[r_{01}, M\left(e, x_{1}, x_{01}\right)\right]=-\left(x_{1} x_{01}\right) r_{01} \in R_{1} \text {. }
$$

Now we expand using the dependence relation and Lemma 3.

$$
\begin{array}{rlr}
{\left[r_{01}, M\left(e, x_{1}, x_{01}\right)\right]=} & -\alpha M\left(\left[r_{01}, e\right], x_{1}, x_{01}\right) & -\alpha M\left(\left[r_{01}, x_{1}\right], e, x_{01}\right) \\
& \alpha M\left(\left[r_{01}, x_{01}\right], e, x_{1}\right) & +\alpha M\left(\left[r_{01}, x_{01}\right], x_{1}, e\right) \\
& (1-\alpha) M\left(\left[e, x_{01}\right], x_{1}, r_{01}\right) & +(1-\alpha) M\left(\left[x_{1}, x_{01}\right], e, r_{01}\right)
\end{array}
$$


Since $R_{10} R_{10} \subseteq R_{01}$ and $R_{01} R_{01} \subseteq R_{10}$, one has $R_{01} \circ R_{01}=0^{* * *}$. One can also show that $0=M\left(R_{01}, e, R_{01}\right)=M\left(R_{10}, e, R_{01}\right)=M\left(R_{10}, e, R_{1}\right)=$ $M\left(R_{10}, R_{1}, e\right)$. Using this we get that

$$
\left[r_{01}, M\left(e, x_{1}, x_{01}\right)\right]=-\alpha M\left(r_{01}, x_{1}, x_{01}\right)-(1-\alpha) M\left(x_{01}, x_{1}, r_{01}\right) .
$$

By (5), this reduces to $(2 \alpha-1) M\left(x_{01}, x_{1}, r_{01}\right)+\alpha M\left(r_{01}, x_{01}, x_{1}\right)$.

$$
\begin{aligned}
M\left(r_{01}, x_{01}, x_{1}\right) & =\left(r_{01}, x_{01}, x_{1}\right)-\left(x_{01}, x_{1}, r_{01}\right) \\
& =-r_{01}^{\circ}\left(x_{01} x_{1}\right)+x_{01}\left(x_{1} r_{01}\right)=0
\end{aligned}
$$

by ${ }^{* * *}$ and $* *$. We have now shown that

$$
\left[r_{01}, M\left(e, x_{1}, x_{01}\right)\right]=(2 \alpha-1) M\left(x_{01}, x_{1}, r_{01}\right) \text {. }
$$

Combining these expressions for $\left[r_{01}, M\left(e, x_{1}, x_{01}\right)\right]$ says

$$
-\left(x_{1} x_{01}\right) r_{01}=(2 \alpha-1) M\left(x_{01}, x_{1}, r_{01}\right)_{1}=(2 \alpha-1)\left(x_{1} x_{01}\right) r_{01} \text {. }
$$

If $\alpha \neq 0$, then $\left(R_{1} R_{01}\right) R_{01}=0$. This says that $R_{1} R_{01}+R_{0} R_{10}$ is an ideal of $R$. By * and ** along with the corresponding results gotten by reversing subscripts, $R_{1} R_{01}+R_{0} R_{10}$ is a trivial ideal.

If $\alpha=0$, the algebra is of type $(0,0,0,0,1)$.

Semiprime. We will show that if $R$ is semiprime, then $M(R, R, R) \subseteq R_{1}+$ $R_{0}$. This will require computing the summands of $M(b, c, d)$ where $b, c, d$ are chosen from the summands in all 64 possible ways. By Lemma 1 and Theorem 6, we can improve our multiplication table for the summands.

THEOREM 7. Let $R$ be a semiprime, right alternative algebra satisfying an identity of form (6). If $R$ has an identity element 1 and an idempotent $e \neq 0, \neq 1$ such that $(e, e, R)=0$, then $R$ has the following multiplication table for the summands.

$\begin{array}{lllll} & & \text { TABLE II } & & \\ & R_{1} & R_{10} & R_{01} & R_{0} \\ R_{1} & R_{1} & R_{10} & 0 & 0 \\ R_{10} & 0 & R_{1}+R_{01} & R_{1} & R_{10} \\ R_{01} & R_{01} & R_{0} & R_{10}+R_{0} & 0 \\ R_{0} & 0 & 0 & R_{01} & R_{0}\end{array}$

If $R$ is semiprime and satisfies $(0,0,0,0,1)$, we have previously shown [5] that $R$ is alternative. In this case $R$ satisfies Table II as well.

In this section we shall assume $R$ satisfies the hypotheses of Theorem 7. We write down all $M(b, c, d)$ where $b, c, d$ are chosen from the summands in all 64 possible ways. The cases $M\left(b_{1}, c_{1}, d_{1}\right) \in R_{1}$ and $M\left(b_{0}, c_{0}, d_{0}\right) \in R_{0}$ are immediately seen to be in $R_{1}+R_{0}$. Of the remaining cases, 38 are zero from Table II and the right alternative law. Of the 24 remaining cases, since 


$$
M(a, b, c)=M(b, a, c),
$$

we really have 16 cases to consider. We examine in detail 8 of the remaining 16; the remainder are gotten by reversing subscripts. In computing the summands for these 8 , we make use of the fact that $a_{10}^{2} \in R_{1}$ and $a_{01}^{2} \in R_{0}$.

We list the 8 cases that we will study.

1. $M\left(R_{1}, R_{10}, R_{10}\right) \subseteq R_{1}+R_{01}$

2. $M\left(R_{10}, R_{10}, R_{1}\right) \subseteq R_{1}$

3. $M\left(R_{0}, R_{10}, R_{10}\right) \subseteq R_{1}+R_{01}$

4. $M\left(R_{10}, R_{10}, R_{0}\right) \subseteq R_{1}+R_{01}$
5. $M\left(R_{10}, R_{10}, R_{01}\right)=0$

6. $M\left(R_{01}, R_{10}, R_{10}\right) \subseteq R_{01}+R_{10}+R_{0}$

7. $M\left(R_{10}, R_{10}, R_{10}\right) \subseteq R_{1}+R_{10}$

8. $M\left(R_{10}, R_{0}, R_{0}\right) \subseteq R_{10}$

The next five lemmas will show that all of these eight alternators lie in $R_{1}+R_{0}$. The algebras $\left(-\frac{1}{2}, 0, \frac{1}{2}, 0, \frac{1}{2}\right)$ and $\left(\frac{1}{2},-\frac{3}{4}, \frac{1}{4}, \frac{1}{4},-\frac{1}{2}\right)$ may be exceptions. It will be extremely useful to remember that each summand absorbs multiplication by $R_{1}$ and $R_{0}$ and that

$$
M(e, R, R)+M(R, e, R)+M(R, R, e) \subseteq R_{1}+R_{0} .
$$

Since we will be using the various equations again and again, we shall tabulate them now for easy reference. The elements $b, c, d$ are intended to be taken from the summands. For $x$, an element in a summand, it is convenient to write $\delta_{x}$ for the number in $\{-1,0,1\}$ such that $[e, x]=\delta_{x} x$. In writing down these equations, we have ignored all alternators containing $e$ as an argument. Thus, the equations should be interpreted to mean equality modulo $M(e, R, R)+M(R, e, R)+M(R, R, e)$. We do not claim

$$
M(e, R, R)+M(R, e, R)+M(R, R, e)
$$

is an ideal; it may be only closed under addition.

\section{TABLE III}

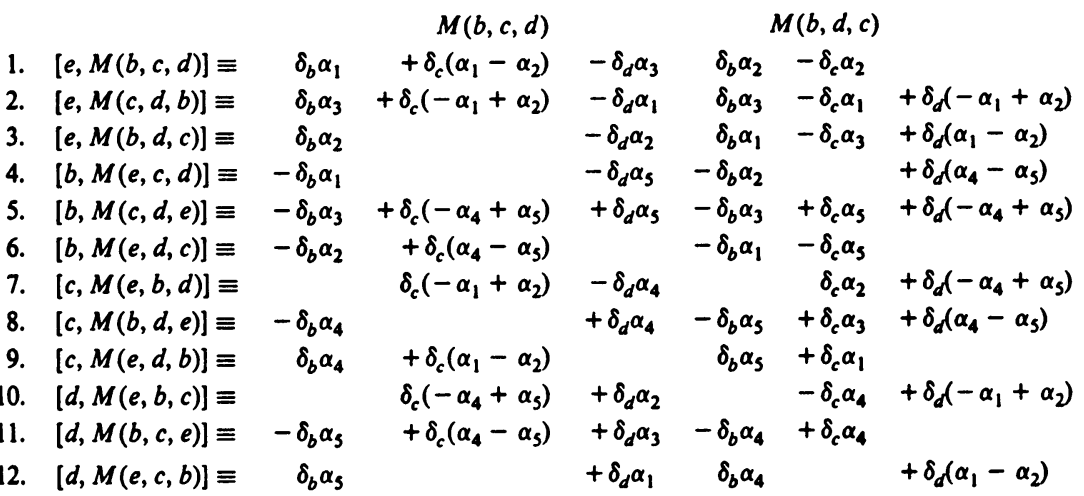

LEMma 4. $M\left(R_{1}, R_{10}, R_{10}\right) \subseteq R_{1}$. 
Proof. Let us consider $b_{10}, c_{10}, d_{1}$ and restrict our attention to the $R_{01}$ component. We already know $M\left(b_{10}, c_{10}, d_{1}\right)_{01}=0$. We shall assume

$$
M\left(b_{10}, d_{1}, c_{10}\right)_{01} \neq 0 \text {. }
$$

The appropriate equations are listed below.

$\begin{array}{rll}\text { Eq. 3. } & {\left[e, M\left(b_{10}, d_{1}, c_{10}\right)\right]_{01} \equiv} & M\left(b_{10}, d_{1}, c_{10}\right)_{01} \\ \text { Eq. 4. } & {\left[b_{10}, M\left(e, c_{10}, d_{1}\right)\right]_{01} \equiv} & -\alpha_{2} \\ \text { Eq. 5. } & {\left[b_{10}, M\left(c_{10}, d_{1}, e\right)\right]_{01} \equiv} & -\alpha_{3}+\alpha_{5} \\ \text { Eq. 6. } & {\left[b_{10}, M\left(e, d_{1}, c_{10}\right)\right]_{01} \equiv} & -\alpha_{1}-\alpha_{5} \\ \text { Eq. 10. } & {\left[d_{1}, M\left(e, b_{10}, c_{10}\right)\right]_{01} \equiv} & -\alpha_{4}\end{array}$

We see that $\alpha_{1}-\alpha_{3}=-1,-\alpha_{2}=0,-\alpha_{3}+\alpha_{5}=0,-\alpha_{1}-\alpha_{5}=0,-\alpha_{4}=$ 0 . The only solution to these five equations is $\left(-\frac{1}{2}, 0, \frac{1}{2}, 0, \frac{1}{2}\right)$. From Lemma 2 we deduce that $R_{10} R_{10} \subseteq R_{01}$ and thus $M\left(b_{10}, d_{1}, c_{10}\right)=-b_{10}{ }^{\circ}\left(d_{1} c_{10}\right)=$ 0 . We have shown that, for any choice of $\alpha_{1}, \alpha_{2}, \alpha_{3}, \alpha_{4}, \alpha_{5}$,

$$
M\left(R_{10}, R_{1}, R_{10}\right)_{01}=0 \text {. }
$$

We have shown $M\left(R_{10}, R_{1}, R_{10}\right) \subseteq R_{1}$; by (5), $M\left(R_{1}, R_{10}, R_{10}\right) \subseteq R_{1}$.

Lemma 5. $M\left(R_{0}, R_{10}, R_{10}\right) \subseteq R_{1} ; M\left(R_{10}, R_{10}, R_{0}\right) \subseteq R_{1}$. The only possible exception is the case $\left(-\frac{1}{2}, 0, \frac{1}{2}, 0, \frac{1}{2}\right)$.

Proof. Let us consider $b_{10}, c_{10}, d_{0}$ and restrict our attention to the $R_{01}$ component. The appropriate equations are listed here.

$M\left(b_{10}, c_{10}, d_{0}\right)_{01} \quad M\left(b_{10}, d_{0}, c_{10}\right)_{01}$

Eq. 1. $\left[e, M\left(b_{10}, c_{10}, d_{0}\right)\right]_{01} \equiv 2 \alpha_{1}-\alpha_{2} \quad 0$

Eq. 3. $\left[e, M\left(b_{10}, d_{0}, c_{10}\right)\right]_{01} \equiv \alpha_{2} \quad \alpha_{1}-\alpha_{3}$

Eq. 4. $\left[b_{10}, M\left(e, c_{10}, d_{0}\right)\right]_{01} \equiv-\alpha_{1} \quad-\alpha_{2}$

Eq. 5. $\left[b_{10}, M\left(c_{10}, d_{0}, e\right)\right]_{01} \equiv-\alpha_{3}-\alpha_{4}+\alpha_{5}-\alpha_{3}+\alpha_{5}$

Eq. 6. $\left[b_{10}, M\left(e, d_{0}, c_{10}\right)\right]_{01} \equiv-\alpha_{2}+\alpha_{4}-\alpha_{5}-\alpha_{1}-\alpha_{5}$

Eq. 7. $\left[c_{10}, M\left(e, b_{10}, d_{0}\right)\right]_{01} \equiv-\alpha_{1}+\alpha_{2} \quad \alpha_{2}$

Eq. 12. $\left[d_{0}, M\left(e, c_{10}, b_{10}\right)\right]_{01} \equiv \alpha_{5}$

$\alpha_{4}$

If we assume $M\left(b_{10}, c_{10}, d_{0}\right)_{01} \neq 0$, then Eq. 1 says $2 \alpha_{1}-\alpha_{2}=-1$, and Eq. $4+$ Eq. 7 says $-2 \alpha_{1}+\alpha_{2}=0$. This contradiction says that

$$
M\left(b_{10}, c_{10}, d_{0}\right)_{01}=0 .
$$

If $M\left(b_{10}, d_{0}, c_{10}\right)_{01} \neq 0$, from Eq. 3 we get $\alpha_{1}-\alpha_{3}=-1$, and the remainder of the listed equations give $\alpha_{2}=0,-\alpha_{3}+\alpha_{5}=0,-\alpha_{1}-\alpha_{5}=0, \alpha_{4}=0$. The only solution to these equations is $\left(-\frac{1}{2}, 0, \frac{1}{2}, 0, \frac{1}{2}\right)$. 
LemMA 6. $M\left(R_{01}, R_{10}, R_{10}\right) \subseteq R_{0}$. The only possible exception is the case $\left(\frac{1}{2}\right.$, $\left.-\frac{3}{4}, \frac{1}{4}, \frac{1}{4},-\frac{1}{2}\right)$.

Proof. We consider $b_{10}, c_{01}, d_{10}$. These are the appropriate equations.

$\begin{array}{lll}\text { Eq. 1. } & {\left[e, M\left(b_{10}, c_{01}, d_{10}\right)\right] \equiv} & \alpha_{2}-\alpha_{3} \\ \text { Eq. 4. } & {\left[b_{10}, M\left(e, c_{01}, d_{10}\right)\right] \equiv} & -\alpha_{1}-\alpha_{5} \\ \text { Eq. 5. } & {\left[b_{10}, M\left(c_{01}, d_{10}, e\right)\right] \equiv} & -\alpha_{3}+\alpha_{4} \\ \text { Eq. 6. } & {\left[b_{10}, M\left(e, d_{10}, c_{01}\right)\right] \equiv} & -\alpha_{2}-\alpha_{4}+\alpha_{5} \\ \text { Eq. 7. } & {\left[c_{01}, M\left(e, b_{10}, d_{10}\right)\right] \equiv} & \alpha_{1}-\alpha_{2}-\alpha_{4}\end{array}$

From Eq. 4, Eq. 5, and Eq. 6 we deduce that $M\left(b_{10}, c_{01}, d_{10}\right)=0$, unless $-\alpha_{1}-\alpha_{5}=0,-\alpha_{3}+\alpha_{4}=0$, and $-\alpha_{2}-\alpha_{4}+\alpha_{5}=0$. This means the algebra is of the type $(\alpha,-\alpha-\beta, \beta, \beta,-\alpha)$. We now examine the individual summands of $M\left(b_{10}, c_{01}, d_{10}\right)$.

If $M\left(b_{10}, c_{01}, d_{10}\right)_{10} \neq 0$, then from Eq. $1, \alpha_{2}-\alpha_{3}=1$ and from Eq. 7 $\alpha_{1}-\alpha_{2}-\alpha_{4}=0$. The algebra is of the type $\left(0, \frac{1}{2},-\frac{1}{2},-\frac{1}{2}, 0\right)$. From Lemma 2 we have $R_{10} R_{10} \subseteq R_{01}$ and $R_{01} R_{01} \subseteq R_{10}$. Thus $M\left(b_{10}, c_{01}, d_{10}\right)=$ $-\left(b_{10} d_{10}\right) \circ c_{01}=0$. This is a contradiction, and we must assume

$$
M\left(R_{10}, R_{01}, R_{10}\right)_{10}=0
$$

If $M\left(b_{10}, c_{01}, d_{10}\right)_{01} \neq 0$, then from Eq. $1, \alpha_{2}-\alpha_{3}=-1$. We examine Eq. 7:

$$
\begin{aligned}
{\left[c_{01}, M\left(e, b_{10}, d_{10}\right)\right] } & =\left[c_{01},-e\left(b_{10} d_{10}\right)\right] \\
& =-c_{01}\left(e \cdot b_{10} d_{10}\right)=-\left(c_{01} \cdot b_{10} d_{10}\right)_{01} \\
& =\left(c_{01}, b_{10}, d_{10}\right)_{01}=M\left(b_{10}, c_{01}, d_{10}\right)_{01}
\end{aligned}
$$

Eq. 7 says that $\alpha_{1}-\alpha_{2}-\alpha_{4}=1$. Thus, the algebra is of the type $\left(\frac{1}{2},-\frac{3}{4}, \frac{1}{4}\right.$, $\left.\frac{1}{4},-\frac{1}{2}\right)$.

We have shown that with one possible exception, $M\left(R_{01}, R_{10}, R_{10}\right) \subseteq R_{0}$.

LEMMA 7. $M\left(R_{10}, R_{10}, R_{10}\right) \subseteq R_{1}$.

Proof. Let us consider $b_{10}, c_{10}, d_{10}$ and restrict our attention to the $R_{10}$ component. These are the appropriate equations. We assume that

$$
M\left(b_{10}, c_{10}, d_{10}\right)_{10} \neq 0
$$


Eq. 1. $\left[e, M\left(b_{10}, c_{10}, d_{10}\right)\right]_{10} \equiv 2 \alpha_{1}-\alpha_{2}-\alpha_{3} \quad 0$

Eq. 4. $\left[b_{10}, M\left(e, c_{10}, d_{10}\right)\right]_{10} \equiv-\alpha_{1}-\alpha_{5} \quad-\alpha_{2}+\alpha_{4}-\alpha_{5}$

Eq. 7. $\left[c_{10}, M\left(e, b_{10}, d_{10}\right)\right]_{10} \equiv-\alpha_{1}+\alpha_{2}-\alpha_{4} \quad \alpha_{2}-\alpha_{4}+\alpha_{5}$

Eq. 10. $\left[d_{10}, M\left(e, b_{10}, c_{10}\right)\right]_{10} \equiv \alpha_{2}-\alpha_{4}+\alpha_{5} \quad-\alpha_{1}+\alpha_{2}-\alpha_{4}$

Eq. 11. $\left[d_{10}, M\left(b_{10}, c_{10}, e\right)\right]_{10} \equiv \alpha_{3}+\alpha_{4}-2 \alpha_{5} \quad 0$

Eq. 12. $\left[d_{10}, M\left(e, c_{10}, b_{10}\right)\right]_{10} \equiv \alpha_{1}+\alpha_{5} \quad \alpha_{1}-\alpha_{2}+\alpha_{4}$

From Eq. $10+$ Eq. $11+$ Eq. $12=0=\left(\alpha_{1}+\alpha_{2}+\alpha_{3}\right) M\left(b_{10}, c_{10}, d_{10}\right)_{10}$, we have $\alpha_{1}+\alpha_{2}+\alpha_{3}=0$. From Eq. 1 we have $M\left(b_{10}, c_{10}, d_{10}\right)_{10}=$ $3 \alpha_{1} M\left(b_{10}, c_{10}, d_{10}\right)_{10}$ and thus $\alpha_{1}=\frac{1}{3}$. Let us examine Eq. 11 .

$$
\begin{aligned}
{\left[d_{10}, M\left(b_{10}, c_{10}, e\right)\right] } & =-\left(b_{10} \circ c_{10}\right) d_{10}=-M\left(b_{10}, c_{10}, d_{10}\right)_{10} \\
& =\left(\alpha_{3}+\alpha_{4}-2 \alpha_{5}\right) M\left(b_{10}, c_{10}, d_{10}\right)_{10}
\end{aligned}
$$

and we have $\alpha_{3}+\alpha_{4}-2 \alpha_{5}=-1$. Now

$$
\begin{aligned}
{\left[b_{10}, M\left(e, c_{10}, d_{10}\right)\right] } & =\left[b_{10},-e\left(c_{10} d_{10}\right)\right]=e\left(c_{10} d_{10}\right) \cdot b_{10} \\
& =\left(c_{10} d_{10} \cdot b_{10}\right)_{10}=\left(c_{10}, d_{10}, b_{10}\right)_{10}=-\left(c_{10}, b_{10}, d_{10}\right)_{10} .
\end{aligned}
$$

Interchanging the letters $b$ and $c$ gives $\left[c_{10}, M\left(e, b_{10}, d_{10}\right)\right]=-\left(b_{10}, c_{10}, d_{10}\right)_{10}$. From Eq. $4+$ Eq. 7 we have

$$
\text { - } M\left(b_{10}, c_{10}, d_{10}\right)_{10}=\left(-2 \alpha_{1}+\alpha_{2}-\alpha_{4}-\alpha_{5}\right) M\left(b_{10}, c_{10}, d_{10}\right)_{10}
$$

and this implies $-2 \alpha_{1}+\alpha_{2}-\alpha_{4}-\alpha_{5}=-1$. Solving these simultaneous equations: $\alpha_{1}+\alpha_{2}+\alpha_{3}=0, \alpha_{1}=\frac{1}{3}, \alpha_{3}+\alpha_{4}-2 \alpha_{5}=-1$, and $-2 \alpha_{1}+\alpha_{2}$ $-\alpha_{4}-\alpha_{5}=-1$ gives us that $M\left(R_{10}, R_{10}, R_{10}\right) \subseteq R_{1}$ unless the algebra is of the type $\left(\frac{1}{3}, \alpha,-\alpha-\frac{1}{3}, \alpha, \frac{1}{3}\right)$.

From the proof of Lemma 2 we know that in this case $\left(x_{10} y_{10}\right)_{1}=\left(y_{10} x_{10}\right)_{1}$ for all $x_{10}, y_{10} \in R_{10}$. We conclude $\left(b_{10} c_{10} \cdot d_{10}\right)_{10}=\left(b_{10} c_{10}\right)_{1} \cdot d_{10}=\left(c_{10} b_{10}\right)_{1}$. $d_{10}=\left(c_{10} b_{10} \cdot d_{10}\right)_{10}=$ by the right alternative law $-\left(c_{10} d_{10} \cdot b_{10}\right)_{10}$. Iterating this three times gives $\left(b_{10} c_{10} \cdot d_{10}\right)_{10}=-\left(b_{10} c_{10} \cdot d_{10}\right)_{10}$. Thus $\left(b_{10} c_{10} \cdot d_{10}\right)_{10}=$ $0=\left(c_{10} b_{10} \cdot d_{10}\right)_{10}$, and thus $M\left(b_{10}, c_{10}, d_{10}\right)_{10}=0$. This contradicts our assumption. We have shown that $M\left(R_{10}, R_{10}, R_{10}\right)_{10}=0$ and so

$$
M\left(R_{10}, R_{10}, R_{10}\right) \subseteq R_{1}
$$

LeMma 8. $M\left(R_{10}, R_{0}, R_{0}\right)=0$.

Proof. We consider $b_{0}, c_{0}, d_{10}$. These are the relevant equations. Notice that $M\left(R_{10}, R_{0}, R_{0}\right) \subseteq R_{10}$; thus, in the equations " $\equiv$ " may be replaced with equality.

$$
\begin{array}{rll} 
& & M\left(b_{0}, d_{10},\right. \\
\text { Eq. 3. } & {\left[e, M\left(b_{0}, d_{10}, c_{0}\right)\right]=} & \alpha_{1}-\alpha_{2} \\
\text { Eq. 10. } & {\left[d_{10}, M\left(e, b_{0}, c_{0}\right)\right]=} & -\alpha_{1}+\alpha_{2}
\end{array}
$$


If $M\left(b_{0}, d_{10}, c_{0}\right) \neq 0$, from Eq. 3 we get $\alpha_{1}-\alpha_{2}=1$. From Eq. 10 we get $-\alpha_{1}+\alpha_{2}=0$. This contradiction implies $M\left(R_{10}, R_{0}, R_{0}\right)=0$.

These last five lemmas give us the following theorem.

THEOREM 8. Let $R$ be a semiprime, right alternative algebra satisfying an identity of form (6). If $R$ has an identity element 1 and an idempotent $e \neq 0, \neq 1$ such that $(e, e, R)=0$, then $M(R, R, R) \subseteq R_{1}+R_{0}$. The only possible exceptions are $\left(-\frac{1}{2}, 0, \frac{1}{2}, 0, \frac{1}{2}\right)$ or $\left(\frac{1}{2},-\frac{3}{4}, \frac{1}{4}, \frac{1}{4},-\frac{1}{2}\right)$.

We intend to show that $M(R, R, R)$ and $R_{10} R_{01}+R_{10}+R_{01}+R_{01} R_{10}$ are ideals which annihilate each other. This will allow us to show that if $R$ is prime, then $R$ is alternative. To this end we prove this lemma.

LeMma 9. $\left(R_{10} R_{10}\right)_{1}$ is an ideal of $R_{1}$.

Proof. It is easy to show that $\left(R_{10} R_{10}\right)_{1}$ is a right ideal of $R_{1}$. To show it is a left ideal requires only that $\left[R_{1},\left(R_{10} R_{10}\right)_{1}\right] \subseteq\left(R_{10} R_{10}\right)_{1}$. Since all

$$
M(e, R, R)_{1}+M(R, e, R)_{1}+M(R, R, e)_{1} \subseteq e\left(R_{10} R_{10}\right),
$$

we may continue to use Table III. Calculating modulo $e\left(R_{10} R_{10}\right)$, we have $M\left(b_{10}, d_{1}, c_{10}\right) \equiv-d_{1} \cdot\left(b_{10} c_{10}\right)$. The relevant equations become:

$\begin{array}{rll} & & M\left(b_{10}, d_{1}, c_{10}\right) \\ \text { Eq. 2. } & {\left[e, M\left(c_{10}, d_{1}, b_{10}\right)\right] \equiv} & -\alpha_{1}+\alpha_{3} \\ \text { Eq. 4. } & {\left[b_{10}, M\left(e, c_{10}, d_{1}\right)\right] \equiv} & -\alpha_{2} \\ \text { Eq. 5. } & {\left[b_{10}, M\left(c_{10}, d_{1}, e\right)\right] \equiv} & -\alpha_{3}+\alpha_{5} \\ \text { Eq. 9. } & {\left[c_{10}, M\left(e, d_{1}, b_{10}\right)\right] \equiv} & \alpha_{1}+\alpha_{5} \\ \text { Eq. 10. } & {\left[d_{1}, M\left(e, b_{10}, c_{10}\right)\right] \equiv} & -\alpha_{4}\end{array}$

Unless $-\alpha_{1}+\alpha_{3}=0,-\alpha_{2}=0,-\alpha_{3}+\alpha_{5}=0, \alpha_{1}+\alpha_{5}=0$, and $-\alpha_{4}=$ 1, we must have $R_{1} \cdot\left(R_{10} R_{10}\right)_{1} \subseteq\left(R_{10} R_{10}\right)_{1}$. Solving these equations simultaneously gives that the algebra is of the type $(0,0,0,-1,0)$. However, in this type, by Lemma 2, $\left(R_{10} R_{10}\right)_{1}=0$. Thus it is always true that $\left(R_{10} R_{10}\right)_{1}$ is an ideal of $R_{1}$.

THEOREM 9. Let $R$ be a semiprime, right alternative algebra satisfying an identity of form (6). If $R$ has an identity element 1 and an idempotent $e \neq 0, \neq 1$ such that $(e, e, R)=0$, then $R$ has an alternative table. The only possible exception is $\left(\frac{1}{2},-\frac{3}{4}, \frac{1}{4}, \frac{1}{4},-\frac{1}{2}\right)$.

Proof. From Theorem 7 we have all products except $R_{10} R_{10}$ and $R_{01} R_{01}$. When $M(R, R, R) \subseteq R_{1}+R_{0}$, it follows that $M(R, R, R) \cdot\left(R_{10}+R_{01}\right)=$ $\left(R_{10}+R_{01}\right) \cdot M(R, R, R)=0$. Since $\left(R_{10} R_{10}\right)_{1} \subseteq M(R, R, R)$, we have from Lemma 9 that $\left(R_{10} R_{10}\right)_{1}$ is an ideal of $R$, and it is easy to see it is trivial. We 
have proved the theorem except for the possible exceptional case when $M(R, R, R)$ may not be contained in $R_{1}+R_{0}$. In the case $\left(-\frac{1}{2}, 0, \frac{1}{2}, 0, \frac{1}{2}\right)$ we know by Lemma 2 that $R_{10} R_{10} \subseteq R_{01}$ and $R_{01} R_{01} \subseteq R_{10}$; in this case the theorem also holds. The last remaining case is $\left(\frac{1}{2},-\frac{3}{4}, \frac{1}{4}, \frac{1}{4},-\frac{1}{2}\right)$.

THEOREM 10. Let $R$ be a prime, right alternative algebra satisfying an identity of the form (6). If $R$ has an identity element 1 and an idempotent $e \neq 0, \neq 1$ such that $(e, e, R)=0$, then $R$ is alternative. The only possible exceptions are $\left(-\frac{1}{2}, 0, \frac{1}{2}, 0, \frac{1}{2}\right)$ and $\left(\frac{1}{2},-\frac{3}{4}, \frac{1}{4}, \frac{1}{4},-\frac{1}{2}\right)$.

Proof. By Theorem 9, $J=R_{10} R_{01}+R_{10}+R_{01}+R_{01} R_{10}$ is an ideal of $R$. By Theorem $8, M(R, R, R) \subseteq R_{1}+R_{0}$ and $M(R, R, R) \cdot\left(R_{10}+R_{01}\right)=$ $\left(R_{10}+R_{01}\right) \cdot M(R, R, R)=0$. It follows that

$$
M(R, R, R) \cdot J=J \cdot M(R, R, R)=0 .
$$

$J=0$ contradicts our assumption that $e \neq 0, \neq 1$. Thus $M(R, R, R)=0$, and $R$ is alternative.

We proved Theorem 9 and Theorem 10 under the assumption that the ring had an identity element. We will now show that the assumption of an identity element is unnecessary. If $R$ is any right alternative algebra over a field $F$, then $R \#=F \times R$ with operations

$$
\begin{gathered}
(\lambda, r)+\left(\lambda^{\prime}, r^{\prime}\right)=\left(\lambda+\lambda^{\prime}, r+r^{\prime}\right), \quad \lambda\left(\lambda^{\prime}, r^{\prime}\right)=\left(\lambda \lambda^{\prime}, \lambda r^{\prime}\right), \\
(\lambda, r)\left(\lambda^{\prime}, r^{\prime}\right)=\left(\lambda \lambda^{\prime}, \lambda r^{\prime}+\lambda^{\prime} r+r r^{\prime}\right)
\end{gathered}
$$

is a right alternative ring. $R$ \# contains an isomorphic copy of $R$ and $(1,0)$ is the identity element of $R \#$. If $R$ is semiprime, then $R \#$ is semiprime. If $R$ satisfies an identity of form (6), then $R$ \# satisfies the same identity. If $e$ is an idempotent of $R$, then $(0, e)$ is an idempotent of $R \#$. If we consider $R \subseteq R$ \#, then the decomposition of $R$ and $R$ \# with respect to an idempotent $e \in R$ satisfies $R_{i j} \subseteq R \#_{i j}$. Theorem 9 implies this corollary.

COROLlaRY. Let $R$ be a semiprime, right alternative algebra satisfying an identity of form (6). If $R$ has an idempotent $e \neq 0, \neq 1$ such that $(e, e, R)=0$, then $R$ has an alternative table. The only possible exception is $\left(\frac{1}{2},-\frac{3}{4}, \frac{1}{4}, \frac{1}{4}\right.$, $-\frac{1}{2}$ ).

We may drop the hypothesis of an identity element from Theorem 10 as well. We need to be more careful because if $R$ is prime, $R$ \# need not be prime. The next corollary follows from the proof of Theorem 10 because when $R$ is prime, either $J \# \cap R$ or $M(R \#, R \#, R \#) \cap R$ is zero.

COROLlARY. Let $R$ be a prime, right alternative algebra satisfying an identity of the form (6). If $R$ has an idempotent $e \neq 0, \neq 1$ such that $(e, e, R)=0$, then 
$R$ is alternative. The only possible exceptions are $\left(-\frac{1}{2}, 0, \frac{1}{2}, 0, \frac{1}{2}\right)$ and $\left(\frac{1}{2},-\frac{3}{4}\right.$, $\left.\frac{1}{4}, \frac{1}{4},-\frac{1}{2}\right)$.

\section{REFERENCES}

1. M. Burrow, Representation theory of finite groups, Academic Press, New York, 1965. MR 38 \#250.

2. H. Celik and D. Outcalt, Power-associativity of antiflexible rings (unpublished).

3. S. Getu and D. Rodabaugh, Generalizing alternative rings, Comm. Algebra 2 (1974), 35-81. MR 50 \# 4682.

4. I. Hentzel, The characterization of $(-1,1)$ rings, J. Algebra 30 (1974), 236-258.

5. I. R. Hentzel and G. M. Piacentini Cattaneo, $A$ note on generalizing alternative rings, Proc. Amer. Math. Soc. 55 (1976), 6-8.

6. M. Humm Kleinfeld, On a class of right alternative rings without nilpotent ideals, J. Algebra 5 (1967), 164-174. MR 35 \#2939.

7. E. Kleinfeld, Right alternative rings, Proc. Amer. Math. Soc. 4 (1953), 939-944. MR 15, 595.

8. E. Kleinfeld, F. Kosier, J. M. Osborn, and D. Rodabaugh, The structure of associator dependent rings, Trans. Amer. Math. Soc. 110 (1964), 473-483. MR 28 \#1221.

9. A.Thedy, Right alternative rings, J. Algebra 37 (1975), 1-43.

Department of Mathematics, Iowa State University, Ames, Iowa 50011

Department of Mathematics, University of Rome, Rome, Italy

Department of Mathematics, Bogazici Universitesi, Istanbul, TURKey 